\title{
Sculpting optical energy landscapes for multi-particle nanoscale assembly
}

\author{
David S. Bradshaw, Kayn A. Forbes and David L. Andrews* \\ School of Chemistry, University of East Anglia, Norwich Research Park, Norwich NR4 7TJ, U. K.
}

\begin{abstract}
To understand the forces and dynamics of two or more neutral particles trapped within an optical beam, careful consideration of the influence of inter-particle forces is required. The well-known, field-independent intrinsic force is known to derive from the Casimir-Polder interaction. However, the magnitude of this force may be over-ridden by the effect known as optical binding, in cases when the laser beam is of sufficient intensity. This binding interaction is completely independent of optomechanical effects relating to optical tweezers, and involves a stimulated (pairwise) forward-scattering process. Unlike the Casimir-Polder coupling, optical binding is not always an attractive force when both particles are in their ground state. Associated with optical binding are potential energy surfaces, which reveal intricate patterns of local minima - sets of positions in which one of the particles will sit at equilibrium (with the other notionally set at the origin). These optical energy landscapes, which can be illustrated by use of contour diagrams, have mostly been considered for systems in which spherical particles are optically bound. The effect of different particle shapes, for example tube-like structures, can also be explored. Moreover, although the theory of conventional optical binding generally assumes situations in which both particles reside in their ground states, new results arise when individual particles are excited to a higher electronic state. Although, in the experimentally most convenient structural configuration (for tumbling spherical particles), pairwise optical binding vanishes in the short-range region, novel effects can arise as a result of non-zero optical binding between three neighbouring particles.
\end{abstract}

Keywords: optical binding, potential energy surfaces, electronic excited states, stimulated forward scattering, nanomanipulation, quantum electrodynamics, optical trapping, Casimir-Polder force

\section{INTRODUCTION}

In general, optical tweezer methods involve individual particles being subjected to an attractive force, usually directed towards the high intensity region of a laser beam. ${ }^{1-4}$ On the inclusion of additional particles within the beam, the positioning and dynamics of each unit becomes subject to inter-particle forces that are distinct and separable from the optomechanical optical tweezer forces. The most familiar intrinsic forces of this inter-particle type are the well-known field-independent Casimir-Polder interactions, ${ }^{5-10}$ which relate to the van der Waals or dispersion interaction, first characterized in London's calculation (which derived an $R^{-6}$ dependence): Casimir and Polder's more accurate derivation, based on quantum electrodynamics (QED), revealed an $R^{-7}$ dependence in the long-range. However, on the presence of a laser beam of sufficient intensity, the form and magnitude of the inter-particle coupling forces may be modified through a phenomenon known as optical binding, ${ }^{11-19}$ which may override the intrinsic force. Despite a diverse set of optical processes being operational for particle sizes approaching or exceeding the wavelength of the throughput beam, the case of non-contact interactions between 'Rayleigh particles' has a specifically QED origin. To be more precise, the optical binding mechanism entails stimulated Rayleigh forward-scattering of the (off-resonant) laser beam, meaning that no net absorption or stimulated emission occurs; the coupling interaction is mediated by virtual photon exchange between the particles (Figure 1). This phenomenon, first predicted using the quantum electrodynamical

Nanophotonics V, edited by David L. Andrews, Jean-Michel Nunzi, Andreas Ostendorf, Proc. of SPIE Vol. 9126, 91260P - ( ) 2014 SPIE · CCC code: 0277-786X/14/\$18 · doi: 10.1117/12.2051207 


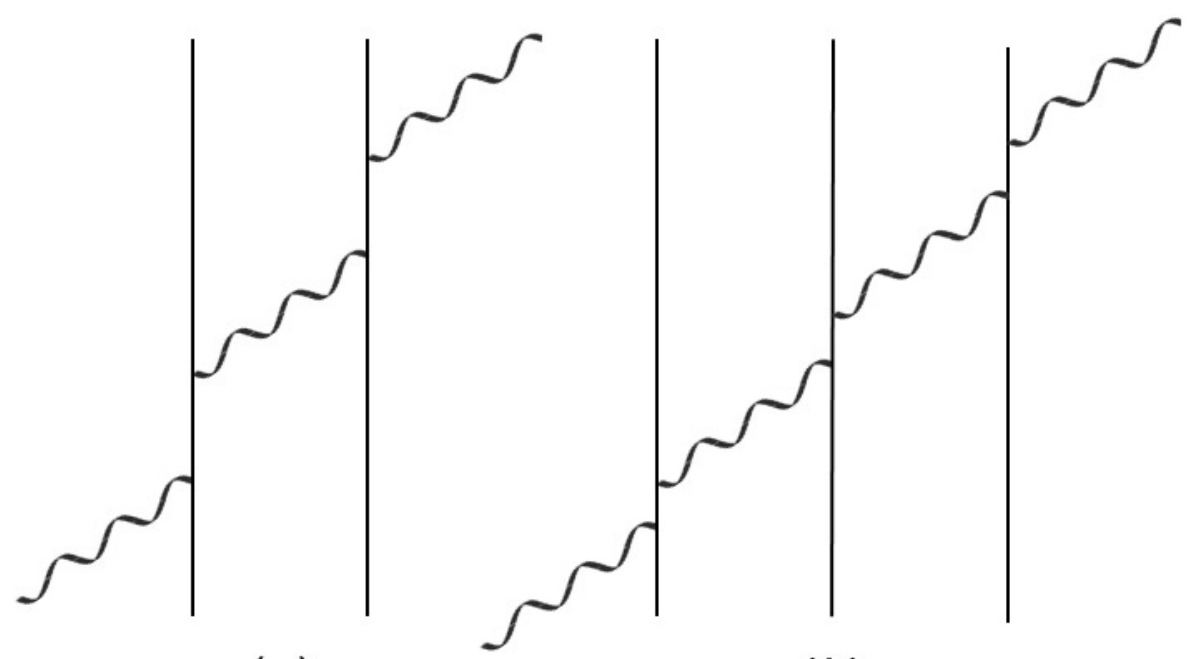

(a)

(b)

Figure 1. Feynman diagrams for optical binding, equivalent to stimulated Rayleigh forward-scattering of an off-resonant laser beam, with time increasing upwards. The two vertical lines denote nanoparticle states and the wavy lines photons: $(a)$ two-particle system, (b) three-particle system.

formalism, ${ }^{20}$ is increasingly advocated as a tool for optical manipulation - indeed, many forms of optically induced array have been observed experimentally. ${ }^{11,21,22}$ In this paper, the quantum electrodynamical theory of optical binding between particles is reviewed and related potential energy surfaces are produced. A brief discussion then follows on the effects of electronically exciting one (or both) of the particles. As an introduction to multi-particle systems, the analysis is finally extended to include three particles with the corresponding optical energy landscapes presented.

\section{THEORY OF OPTICAL BINIDING}

\subsection{General expression}

To properly account for the quantum features arising for optical binding between Rayleigh particles, the Power-ZienauWoolley approach is employed. ${ }^{23}$ Using this framework, the multipolar Hamiltonian, $H$, for a pair of nanoparticles $A$ and $B$ is represented as;

$$
H=\sum_{\xi=A, B} H_{\text {part }}(\xi)+\sum_{\xi=A, B} H_{\text {int }}(\xi)+H_{\text {rad }},
$$

where $H_{\text {part }}(\xi)$ is the Hamiltonian for nanoparticle $\xi$ and $H_{\text {rad }}$ denotes the energy operator for the radiation field. The Hamiltonian $H_{\text {int }}(\xi)$ gives the interaction of the field with $\xi$ and, using the electric dipole approximation, the following applies;

$$
H_{\text {int }}(\xi)=-\varepsilon_{0}^{-1} \sum_{\xi} \boldsymbol{\mu}(\xi) \cdot \mathbf{d}^{\perp}\left(\mathbf{R}_{\xi}\right)
$$


with $\boldsymbol{\mu}(\xi)$ and $\mathbf{R}_{\xi}$ as the electric-dipole moment operator and the position vector, respectively. The operator $\mathbf{d}^{\perp}\left(\mathbf{R}_{\xi}\right)$ represents the transverse electric displacement field of the radiation. General results for the optical binding forces between a pair of neutral nanoparticles $A$ and $B$ can be determined from an expression for the distance-dependent energy shift, $\Delta E$, which in turn is obtained by the application of fourth-order perturbation theory, i.e.;

$$
\Delta E=\operatorname{Re}\left\{\sum_{t, s, r} \frac{\left\langle i\left|H_{\mathrm{int}}\right| t\right\rangle\left\langle t\left|H_{\mathrm{int}}\right| s\right\rangle\left\langle s\left|H_{\mathrm{int}}\right| r\right\rangle\left\langle r\left|H_{\mathrm{int}}\right| i\right\rangle}{\left(E_{i}-E_{t}\right)\left(E_{i}-E_{s}\right)\left(E_{i}-E_{r}\right)}\right\},
$$

where $|i\rangle$ is the unperturbed state of the system comprising the molecules and the radiation field, in which both $A$ and $B$ are in their individual electronic ground states; $|r\rangle,|s\rangle$ and $|t\rangle$ are virtual system states, and $E_{n}$ is the energy of state $|n\rangle$. The system states can be written in the form;

$$
|n\rangle=\left|\operatorname{part}_{n}\right\rangle\left|\operatorname{rad}_{n}\right\rangle \equiv\left|\operatorname{part}_{n} ; \operatorname{rad}_{n}\right\rangle,
$$

with $\left|\operatorname{part}_{n}\right\rangle$ and $\left|\operatorname{rad}_{n}\right\rangle$ defining the status of all nanoparticle and radiation states, respectively. The laser-induced interaction entails the annihilation of a 'real' (laser throughput) photon at one particle and the stimulated emission of a 'real' photon at the other, with a virtual photon mediating the coupling between them; this results in elastic forwardscattering of the off-resonant beam as shown by Figure 1 $(a)$. The nanoparticles and the throughput radiation suffer no overall change in quantum state. Using the implied summation convention for repeated Cartesian tensor subscript indices, as shown previously, the short-range result for $\Delta E$ emerges as $;^{24}$

$$
\begin{aligned}
\Delta E & =\left(\frac{I}{2 \varepsilon_{0} c}\right) e_{i} \bar{e}_{l} \operatorname{Re}\left\{\alpha_{i j}^{A}(k) V_{j k}(k, \mathbf{R}) \alpha_{k l}^{B}(k) \mathrm{e}^{-i \mathbf{k} \cdot \mathbf{R}}+\alpha_{i j}^{B}(k) V_{j k}(k, \mathbf{R}) \alpha_{k l}^{A}(k) \mathrm{e}^{i \mathbf{k} \cdot \mathbf{R}}\right. \\
& \left.+\beta_{i j l}^{A}(k) V_{j k}(0, \mathbf{R}) \mu_{k}^{B}+\mu_{k}^{A} V_{j k}(0, \mathbf{R}) \beta_{i j l}^{B}(k)\right\},
\end{aligned}
$$

where $I$ is the intensity of the input laser beam, $V_{j k}$ is the dipole-dipole coupling tensor ${ }^{25,26}$ (the first two terms corresponding to a dynamic interaction, as shown by the dependence on $k$, and the final two terms are static), $\hbar c k$ is the energy per photon, $\mathbf{e}$ is the polarization of the input beam; $\alpha_{i j}^{(\xi)}(k)$ and $\beta_{i j l}^{(\xi)}(k)$ represent the polarizability and hyperpolarizability tensor, respectively, and $\mu^{\xi}$ is a permanent dipole moment. The optical binding force is determined from equation (5) via the expression $\mathbf{F}=-\partial \Delta E / \partial \mathbf{R}$, the result of which is found to have an $R^{-4}$ distance dependence in the short-range. For spherical particles (the main subject of this paper), which necessarily contain no permanent dipoles, only the first two terms of equation (5) arise. In contrast, all four terms are required for cylindrical structures, such as carbon nanotubes, with a static dipole - as outlined in the following sub-section.

\subsection{Expression for two parallel tube-like structures}

We briefly recap on a system involving nanoparticles of cylindrical symmetry configured in a parallel arrangement. In contrast to the case of spherical particles there are five degrees of freedom for the spatial and orientation disposition of such a system, relative to the salient field vectors (namely, the propagation and polarization vectors). In detail, we can describe a pair of parallel nanoparticles oriented against a system of axes such that each axis is aligned with the $x$-axis and the displacement, $\mathbf{R}$, between the nanoparticles lies on the $z$-axis. The assumed plane-polarized throughput radiation is defined through the angles $\phi$ and $\theta$ made by its $\mathbf{e}$ vector against $\mathbf{R}$ in the $x z$-plane, and the particle axis in the $x y$-plane, respectively. By expressing the polarization unit vector in the cylindrical form $\mathbf{e}=\cos \phi \mathbf{i}+\sin \phi \sin \theta \mathbf{j}+\sin \phi \cos \theta \mathbf{k}$, and employing the explicit form of the $V_{j k}$ tensor, the general expression (5) gives; 


$$
\begin{aligned}
\Delta E & =\left(\frac{I}{4 \pi \varepsilon_{0}^{2} c}\right)\left[\left\{\left(\alpha_{\perp}^{2} \sin ^{2} \phi \sin ^{2} \theta+\alpha_{\|}^{2} \sin ^{2} \phi \cos ^{2} \theta\right)\left(\frac{\cos k R}{R^{3}}+\frac{k \sin k R}{R^{2}}-\frac{k^{2} \cos k R}{R}\right)\right.\right. \\
& \left.\left.-2 \alpha_{\perp}^{2} \cos ^{2} \phi\left(\frac{\cos k R}{R^{3}}+\frac{k \sin k R}{R^{2}}\right)\right\} \cos (\mathbf{k} \cdot \mathbf{R})+\frac{\mu_{\|}}{R^{3}}\left(\beta_{\perp_{2}}\left(\sin ^{2} \phi \sin ^{2} \theta+\cos ^{2} \phi\right)+\beta_{\|} \sin ^{2} \phi \cos ^{2} \theta\right)\right],
\end{aligned}
$$

where $\alpha_{y y}=\alpha_{z z} \equiv \alpha_{\perp}, \alpha_{x x} \equiv \alpha_{\|}$and $\beta_{x x x}=\beta_{\|}, \beta_{y x y}=\beta_{z x z}=\beta_{\perp_{2}}$. Again using $\mathbf{F}=-\partial \Delta E / \partial \mathbf{R}$, in the short-range region, equation (6) is now written as;

$$
F_{z}=\left(\frac{3 I \hat{R}_{z}}{4 \pi \varepsilon_{0}^{2} c R^{4}}\right)\left(\left(\alpha_{\|}^{2}+\mu_{\|}\left(\beta_{\|}-\beta_{\perp_{2}}\right)\right) \sin ^{2} \phi \cos ^{2} \theta+\mu_{\|} \beta_{\perp_{2}}+\alpha_{\perp}^{2}\left(\sin ^{2} \phi\left(3-\cos ^{2} \theta\right)-2\right)\right),
$$

which represents an optical binding force in the $z$-direction. To determine typical magnitudes for this force, we employ carbon nanotubes of length $200 \mathrm{~nm}$ and $0.4 \mathrm{~nm}$ in radius, and position them $2 \mathrm{~nm}$ apart. On the assumption that the $\alpha_{\perp}$ and $\alpha_{\|}$values are consistent with the static polarizabilities of the nanotubes, ${ }^{25}$ equation (7) gives a repulsive force in the $\mu \mathrm{N}$ range for $\phi=0^{\circ}$ (independent of $\theta$ ), and an attractive force in the $p \mathrm{~N}$ range for $\phi=90^{\circ}$ (the value of $\theta$ has a very small effect). ${ }^{26}$ For spherical particles, the condition $\alpha_{\perp}=\alpha_{\|}=\alpha$ is imposed and $\theta$ equates to null, so that equation (7) becomes;

$$
F_{z}=\left(\frac{3 I \hat{R}_{z} \alpha^{2}}{4 \pi \varepsilon_{0}^{2} c R^{4}}\right)\left(3 \sin ^{2} \phi-2\right)
$$

on isotropically averaging the system with respect to the incoming light the optical binding force will disappear, i.e. $\left\langle F_{z}\right\rangle=0$ since $\left\langle\sin ^{2} \theta\right\rangle=2 / 3$; in such a tumbling configuration pairwise interactions are negated.

\subsection{Optical binding modification via nanoparticle excitation}

In the previous section, our considerations of optical binding have been based on the notion that both nanoparticles reside in their ground state. Here, we consider the effects that may arise when one of the particles becomes electronically excited: the condition is achievable either through direct photo-excitation (with the same beam as is responsible for the optical manipulation, or another that traverses it) or resonance energy transfer. Although, the Casimir-Polder interaction is itself negligible compared to optical binding, given the input of a beam with sufficient intensity, it is interesting to observe that the dispersion force is not always attractive when one (or more) particles are excited, as detailed quantum electrodynamical analysis has shown. ${ }^{27,28}$

Returning to optical binding, Figure 2 visually shows that optomechanical motion is possible between the particle pair through manipulation of the optical binding - this is achieved by excitation of either particle. Moving across the diagram, $A$ is excited through one-photon absorption (energy transfer from a nearby non-interacting particle is also possible) - resulting in modification to the pair-potential energy. Moving to the right-hand side of the diagram, resonance energy transfer between the pair is represented. It is noteworthy that, due to the presence of the off-resonant beam, the efficiency of resonant absorption and resonance energy transfer may be also be subjected to significant modification in such a scheme. ${ }^{28}$ A much more detailed analysis on such a scheme is found within an earlier proceedings paper. ${ }^{29}$ 


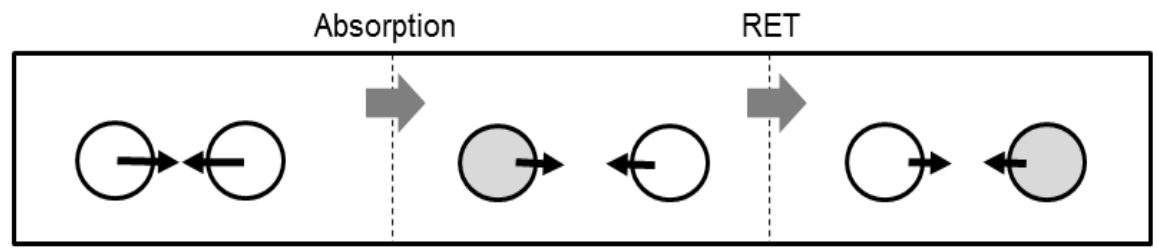

(a)

(b)

(c)

Figure 2. Representation of optical binding for: $(a) A$ and $B$ both in their ground states (unfilled circles), $(b) A$ in an excited state (filled circle) with $B$ remaining unexcited, and $(c) A$ unexcited and $B$ excited. The successive states are achievable through the sequential optical process, shown horizontally across the diagram, of one-photon absorption (first block arrow) followed by resonance energy transfer (second block arrow). The black arrows on the nanoparticles denote arbitrary changes in the optical binding force.

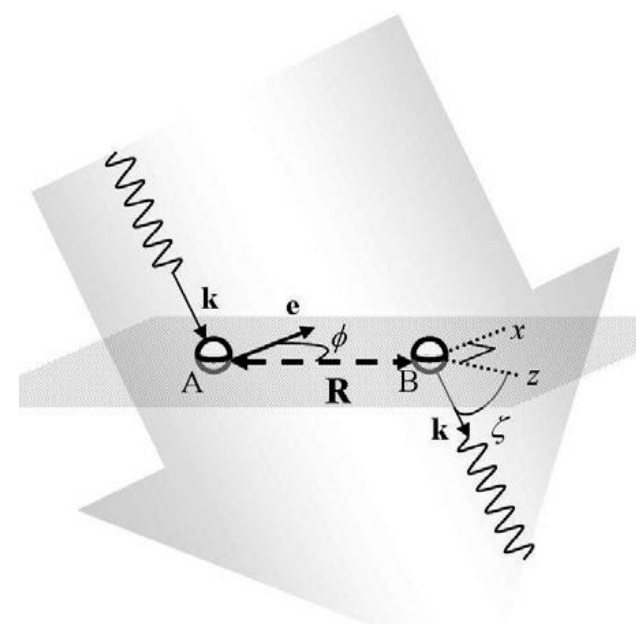

Figure 3. Nanoparticles $A$ and $B$, residing in the $x$, $z$-plane and displaced by $\mathbf{R}$, are trapped in an off-resonant laser beam that is linearly polarized. The polarization vector, e, points in the $x$-direction, forming an angle $\phi$ with $\mathbf{R}$, the beam propagation vector $\mathbf{k}$ creates an angle $\zeta$ with the $x$, $z$-plane.

\section{POTENTIAL ENERGY SURFACES}

\subsection{Two-particle system}

Using equation (5) as a starting point, the following energy shift expression is found (for spherical particles) as a function of the geometric parameters shown in Figure 3; $;^{30,31}$

$$
\Delta E=\left(\frac{2 I}{\varepsilon_{0} c}\right) e_{i} \bar{e}_{l} \operatorname{Re}\left\{\alpha^{A}(k) V_{x x}(k, \mathbf{R}) \alpha^{B}(k)\right\} \cos (k R \sin \phi \cos \zeta)
$$

where $\alpha^{\xi}(k)$ is a scalar polarizability. If cylindrical particles are again considered, additional degrees of freedom are necessary to describe the orientation of the particle axes. However, the largest polarizability component of each nanoparticle is expected to be aligned with the polarization of the beam, if the particles are well-separated. Under these conditions, only the diagonal (principal axis) elements of the polarizability tensor, on the framework illustrated in Figure 3 , need be considered in the summation of equation (5). Moreover, due to the orientation of the polarization on the $x$-axis, only one term will contribute. In consequence, the energy shift expression for cylindrical nanoparticles is again 


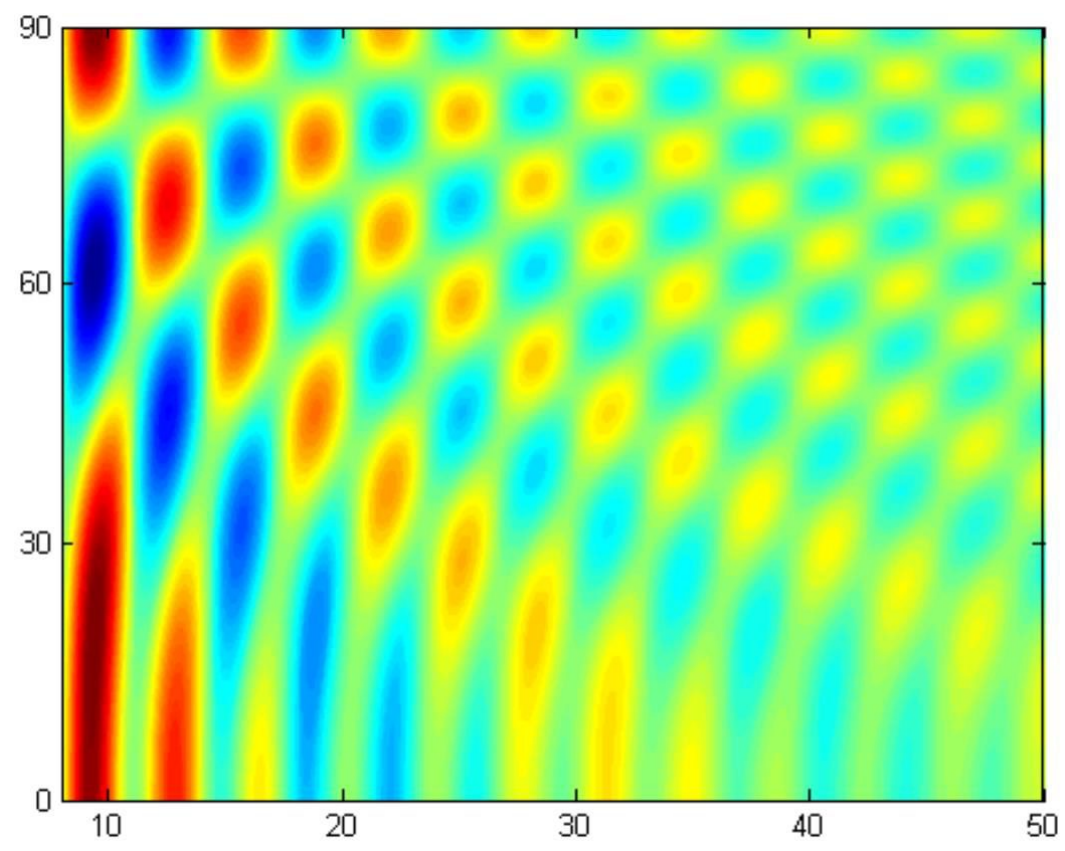

Figure 4. Contour map of the optically binding energy shift for two particles: $\Delta E$ is plotted against $\zeta$ and $k R$, and the angle $\varphi$ is fixed at $\pi / 4$. Adjacent, differently colored islands signify alternating local maxima (red) and minima (blue).

delivered by equation (9), although $\alpha^{A}(k)$ and $\alpha^{B}(k)$ are now interpreted as the largest polarizability elements of the particles $A$ and $B$ respectively - usually corresponding to the length-axis of the cylinder. A potential energy surface for optical binding, as shown in Figure 4, is obtained from equation (9). This contour diagram contains information on the location of the stability points of the binding system, i.e. the set of positions where one of the nanoparticles will sit at equilibrium (with the other notionally set at the origin). Moreover, a particle positioned away from the minima will experience forces directed towards the nearest points of stability.

\subsection{Three-particle system}

The two-particle system is now extended to a set of three nanoparticles. Along with the pairwise optical binding mechanism described previously, three body interactions - which are illustrated by Figure $1(b)$ - should also be considered in such a case, i.e.;

$$
\Delta E=\Delta E_{B A}+\Delta E_{C A}+\Delta E_{C B}+\Delta E_{A B C},
$$

in which $\triangle E_{A B C}$ is the energy shift due to three-body coupling. Explicitly, the latter is defined generally by;

$$
\begin{aligned}
\Delta E_{A B C}= & \left(\frac{I}{2 \varepsilon_{0} c}\right) e_{i} \bar{e}_{l}\left\{\alpha_{i j}^{A}(k) V_{j k}\left(k, \mathbf{R}_{B A}\right) \alpha_{k l}^{B}(k) V_{l m}\left(k, \mathbf{R}_{C B}\right) \alpha_{m n}^{C}(k) \cos \left(\mathbf{k} \cdot \mathbf{R}_{C A}\right)+\alpha_{i j}^{A}(k) V_{j k}\left(k, \mathbf{R}_{B A}\right) \alpha_{k l}^{C}(k) V_{l m}\left(k, \mathbf{R}_{C B}\right)\right. \\
& \left.\times \alpha_{m n}^{B}(k) \cos \left(\mathbf{k} \cdot \mathbf{R}_{B A}\right)+\alpha_{i j}^{B}(k) V_{j k}\left(k, \mathbf{R}_{B A}\right) \alpha_{k l}^{A}(k) V_{l m}\left(k, \mathbf{R}_{C B}\right) \alpha_{m n}^{C}(k) \cos \left(\mathbf{k} \cdot \mathbf{R}_{C B}\right)\right\},
\end{aligned}
$$


where $\mathbf{R}_{\xi_{1} \xi_{2}}=\mathbf{R}_{\xi_{1}}-\mathbf{R}_{\xi_{2}}$, with $\xi_{1}$ and $\xi_{2}$ representing either particle $A, B$ or $C$. To produce optical energy landscapes for the three-particle configuration, a simple geometry is chosen in which the spherical particles are positioned in a straight line, as shown by Figure 5. Employing this model, equation (11) becomes;

$$
\begin{aligned}
\Delta E_{A B C}= & \left(\frac{I \alpha^{A} \alpha^{B} \alpha^{C}}{16 \pi^{2} \varepsilon_{0}^{3} c R_{B A}^{3} R_{C B}^{3}}\right)\left(\left(1-k^{2} R_{B A}^{2}\right) \cos \left(k R_{B A}\right)+k R_{B A} \sin \left(k R_{B A}\right)\right)\left(\left(1-k^{2} R_{C B}^{2}\right) \cos \left(k R_{C B}\right)+k R_{C B} \sin \left(k R_{C B}\right)\right) \\
& \left(\cos \left(k R_{C A}\right)+\cos \left(k R_{B A}\right)+\cos \left(k R_{C B}\right)\right),
\end{aligned}
$$

where the dependence on the polarizability is suppressed. Moreover, the pairwise optical binding is written as;

$$
\Delta E_{\xi_{1} \xi_{2}}=\left(\frac{I k^{2} \alpha^{\xi_{1}} \alpha^{\xi_{2}}}{4 \pi \varepsilon_{0}^{2} c R_{\xi_{1} \xi_{2}}^{3}}\right)\left(\cos ^{2} k R_{\xi_{1} \xi_{2}}+k R_{\xi_{1} \xi_{2}} \sin k R_{\xi_{1} \xi_{2}} \cos k R_{\xi_{1} \xi_{2}}-k^{2} R_{\xi_{1} \xi_{2}}^{2} \cos ^{2} k R_{\xi_{1} \xi_{2}}\right),
$$

in which $\Delta E_{\xi_{1} \xi_{2}}$ denotes either $\Delta E_{B A}, \Delta E_{C B}$ or $\Delta E_{C A}$ as follows from equation (10). The optical energy landscape for three particles, in a configuration given by Figure 5, is constructed from equation (10) following insertion of equations (12) and (13). The corresponding contour map of $\Delta E$ against $k R_{B A}$ and $k R_{C B}$ is shown in Figure 6; here, we assume that the volume polarizabilities, $\alpha / 4 \pi \varepsilon_{0}$, are similar for each molecule and approximately equal to the molecular volume.

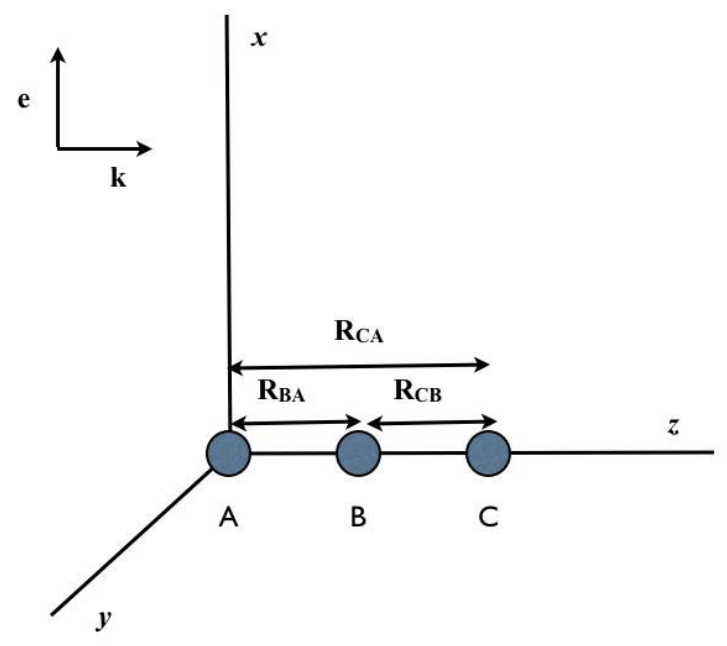

Figure 5. Geometry for a system containing three spherical nanoparticles. The wave-vector, $\mathbf{k}$, of the input radiation is directed parallel to $\mathbf{R}_{\xi_{1} \xi_{2}}=\mathbf{R}_{\xi_{1}}-\mathbf{R}_{\xi_{2}}$, where $\xi_{1}$ and $\xi_{2}$ represent either particle $A, B$ or $C$.

\section{CONCLUSION}

The aim of this paper has been to detail the generation of optical energy landscapes, corresponding to optical binding, for a two and three nanoparticle configuration. In addition, it has been demonstrated that a modification to the optical binding potential is possible on excitation of an interacting particle, and typical values for the optical binding force between nanotubes have been reported. In ongoing and future research, we intend to add more particles to the threeparticle system - where an especially interesting case is that of optical binding between multiple particles in a threedimensional geometry. This analysis should provide a consolidated theoretical basis for understanding the mechanisms at work in studies of optically induced self-assembly by nanoparticles - itself a subject of highly active experimental investigation. 


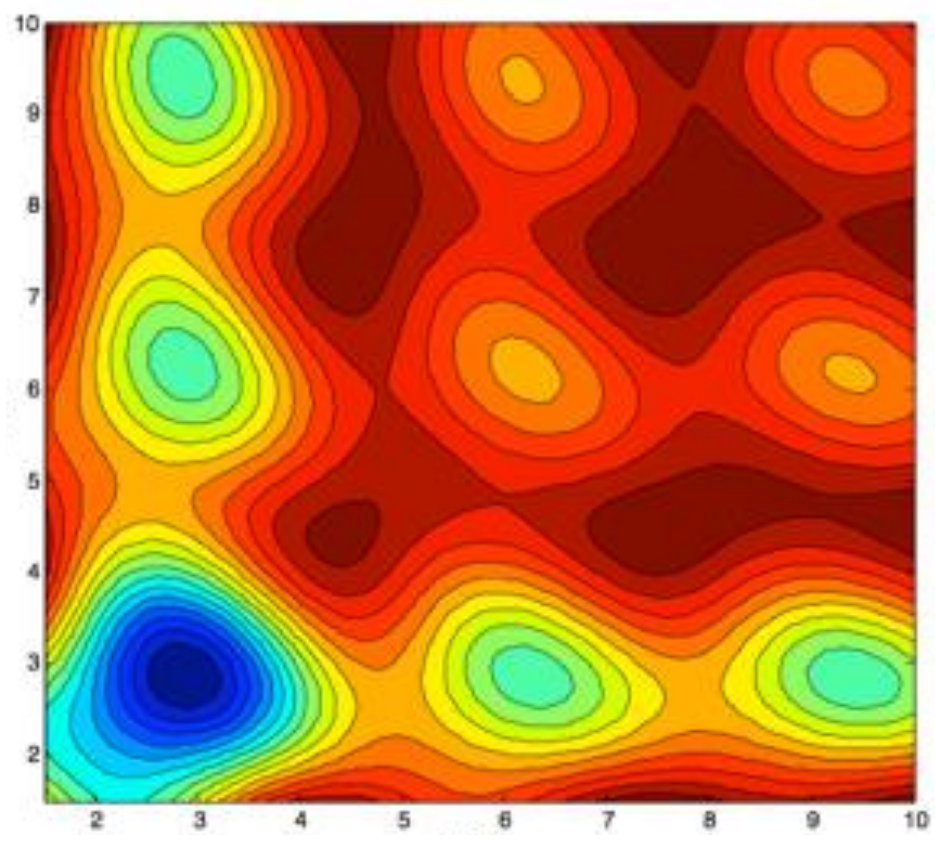

Figure 6. Contour map of the optically binding energy shift for three particles: $\Delta E$ is plotted against $k R_{B A}$ and $k R_{C B}$. In this graph the three spherical nanoparticles have a radius of $10 \mathrm{~nm}$ and the input laser wavelength is $562 \mathrm{~nm}$.

\section{ACKNOWLEDGMENTS}

DSB and DLA are grateful to the Leverhulme Trust for funding our research.

\section{REFERENCES}

[1] Molloy, J. E. and Padgett, M. J., "Lights, action: optical tweezers," Contemp. Phys. 43, 241-258 (2002).

[2] Neuman, K. C. and Nagy, A., "Single-molecule force spectroscopy: optical tweezers, magnetic tweezers and atomic force microscopy," Nature Meth. 5, 491-505 (2008).

[3] Juan, M. L., Righini, M. and Quidant, R., "Plasmon nano-optical tweezers," Nat. Photonics 5, 349-356 (2011).

[4] Jones, P. H., [Optical tweezers] Taylor \& Francis, New York(2013).

[5] Cohen, A. E. and Mukamel, S., "Resonant enhancement and dissipation in nonequilibrium van der Waals forces," Phys. Rev. Lett. 91, 233202 (2003).

[6] Lamoreaux, S. K., "The Casimir force: background, experiments, and applications," Rep. Prog. Phys. 68, 201-236 (2005).

[7] Obrecht, J. M., Wild, R. J., Antezza, M., Pitaevskii, L. P., Stringari, S. and Cornell, E. A., "Measurement of the temperature dependence of the Casimir-Polder force," Phys. Rev. Lett. 98, 233202 (2007).

[8] Buhmann, S. Y. and Scheel, S., "Thermal Casimir versus Casimir-Polder forces: Equilibrium and nonequilibrium forces," Phys. Rev. Lett. 100, 253201 (2008).

[9] Rodríguez, J. and Salam, A., "Casimir-Polder potential in a dielectric medium out of thermal equilibrium," Phys. Rev. A 82, 062522 (2010).

[10] Rodríguez, J. and Salam, A., "On the role of dissipation on the Casimir-Polder potential between molecules in dielectric media," J. Chem. Phys. 133, 164501 (2010).

[11] Mohanty, S. K., Andrews, J. T. and Gupta, P. K., "Optical binding between dielectric particles," Opt. Express 12, 2746-2753 (2004). 
[12] Haefner, D., Sukhov, S. and Dogariu, A., "Conservative and nonconservative torques in optical binding," Phys. Rev. Lett. 103, 173602 (2009).

[13] Taylor, J. M. and Love, G. D., "Optical binding mechanisms: A conceptual model for Gaussian beam traps,” Opt. Express 17, 15381-15389 (2009).

[14] Brzobohatý, O., Čižmár, T., Karásek, V., Šiler, M., Dholakia, K. and Zemánek, P., "Experimental and theoretical determination of optical binding forces," Opt. Express 18, 25389-25402 (2010).

[15] Čižmár, T., Dávila Romero, L. C., Dholakia, K. and Andrews, D. L., "Multiple optical trapping and binding: New routes to self-assembly,” J. Phys. B: At. Mol. Opt. Phys. 43, 102001 (2010).

[16] Dholakia, K. and Zemanek, P., "Gripped by light: Optical binding," Rev. Mod. Phys. 82, 1767-1791 (2010).

[17] Salam, A., "Molecular quantum electrodynamics of radiation-induced intermolecular forces," Adv. Quant. Chem. 62, 1-34 (2011).

[18] Demergis, V. and Florin, E.-L., "Ultrastrong optical binding of metallic nanoparticles," Nano Lett. 12, 5756-5760 (2012).

[19] Lemeshko, M. and Friedrich, B., "Interaction between polar molecules subject to a far-off-resonant optical field: entangled dipoles up- or down-holding each other," Mol. Phys. 110, 1873-1881 (2012).

[20] Thirunamachandran, T., "Intermolecular interactions in the presence of an intense radiation field," Mol. Phys. 40, 393-399 (1980).

[21] Tatarkova, S. A., Carruthers, A. E. and Dholakia, K., "One-dimensional optically bound arrays of microscopic particles," Phys. Rev. Lett. 89, 283901 (2002).

[22] Mellor, C. D., Fennerty, T. A. and Bain, C. D., "Polarization effects in optically bound particle arrays," Opt. Express 14, 10079-10088 (2006).

[23] Woolley, R. G., "Gauge invariance in non-relativistic electrodynamics," Proc. R. Soc. A 456, 1803-1819 (2000).

[24] Bradshaw, D. S. and Andrews, D. L., "Optically induced forces and torques: Interactions between nanoparticles in a laser beam," Phys. Rev. A 72, 033816 (2005).

[25] Guo, G. Y., Chu, K. C., Wang, D.-S. and Duan, C.-G., "Static polarizability of carbon nanotubes: ab initio independent-particle calculations," Comput. Mater. Sci. 30, 269-273 (2004).

[26] Andrews, D. L. and Bradshaw, D. S., "Laser-induced forces between carbon nanotubes," Opt. Lett. 30, 783-785 (2005).

[27] Andrews, D. L., Bradshaw, D. S., Leeder, J. M. and Rodríguez, J., "Dynamics of the dispersion interaction in an energy transfer system," Phys. Chem. Chem. Phys. 10, 5250-5255 (2008).

[28] Bradshaw, D. S. and Andrews, D. L., "Interparticle interactions: Energy potentials, energy transfer, and nanoscale mechanical motion in response to optical radiation," J. Phys. Chem. A 117, 75-82 (2013).

[29] Bradshaw, D. S. and Andrews, D. L., "Near-field manipulation of interparticle forces through resonant absorption, optical binding, and dispersion forces," Proc. SPIE 8810, 881025 (2013).

[30] Rodríguez, J., Davila Romero, L. C. and Andrews, D. L., "Optically induced potential energy landscapes," J. Nanophoton. 1, 019503 (2007).

[31] Dávila Romero, L. C., Rodríguez, J. and Andrews, D. L., "Electrodynamic mechanism and array stability in optical binding," Opt. Commun. 281, 865-870 (2008).

*david.andrews@physics.org 